\title{
Turbulent Boundary Layer in High Rayleigh Number Convection in Air
}

\author{
Ronald du Puits, Ling Li, Christian Resagk, and André Thess \\ Technische Universitaet Ilmenau, Institute of Thermodynamics and Fluid Mechanics, P.O. Box 100565, 98684 Ilmenau, Germany \\ Christian Willert \\ German Aerospace Center, Institute of Propulsion Technology, 51170 Koeln, Germany
}

(Received 20 June 2013; published 26 March 2014)

\begin{abstract}
Flow visualizations and particle image velocimetry measurements in the boundary layer of a RayleighBénard experiment are presented for the Rayleigh number $\mathrm{Ra}=1.4 \times 10^{10}$. Our visualizations indicate that the appearance of the flow structures is similar to ordinary (isothermal) turbulent boundary layers. Our particle image velocimetry measurements show that vorticity with both positive and negative sign is generated and that the smallest flow structures are 1 order of magnitude smaller than the boundary layer thickness. Additional local measurements using laser Doppler velocimetry yield turbulence intensities up to $I=0.4$ as in turbulent atmospheric boundary layers. From our observations, we conclude that the convective boundary layer becomes turbulent locally and temporarily although its Reynolds number $\operatorname{Re} \approx 200$ is considerably smaller than the value 420 underlying existing phenomenological theories. We think that, in turbulent Rayleigh-Bénard convection, the transition of the boundary layer towards turbulence depends on subtle details of the flow field and is therefore not universal.
\end{abstract}

DOI: 10.1103/PhysRevLett.112.124301

PACS numbers: 44.25.+f, 47.20.Bp, 47.20.Ib

Rayleigh-Bénard convection (RBC) in a fluid layer heated from below and cooled from above is a canonical problem of turbulence research. Its understanding is crucial for the accurate prediction of heat transfer in applications as diverse as room ventilation [1], solar convection [2], and ocean circulation [3]. Although RBC has been comprehensively investigated over the past two decades [4-7], our understanding of the transition to the "ultimate regime" [8] at very high Rayleigh numbers [9-12] is still incomplete. This is partially due to the lack of knowledge of under which conditions the boundary layers at the heating and cooling plates undergo a transition from laminar to turbulent flow. The present experimental work intends to shed new light on this fundamental question. More precisely, we demonstrate that the convective layer displays features of turbulence significantly below the expected threshold.

It is commonly accepted $[8,13,14]$ that the transition to the ultimate regime where the Nusselt number $\mathrm{Nu}$ scales with the Rayleigh number $\mathrm{Ra}$ as $\mathrm{Nu} \sim \mathrm{Ra}^{1 / 2}$ takes place when the boundary layers become turbulent. It is often argued that this transition takes place when the boundarylayer Reynolds number (to be defined below) exceeds a value of approximately $\operatorname{Re}_{c}=320$ [9] or $\operatorname{Re}_{c}=420$ [14] depending on the model underlying these numbers. However, this figure cannot be expected to be a reliable prediction for the transition to turbulence in the convective boundary layer of RBC for at least three reasons. (i) The often-cited threshold $\operatorname{Re}_{c}=420$ was derived in Refs. $[15,16]$ under the assumption that the horizontal velocity is described by the Blasius profile of an isothermal flat-plate boundary layer. In reality, however, the convective boundary layer of RBC is affected by buoyancy forces $[17,18]$ and can deviate significantly from the Blasius shape [19-22]. (ii) The threshold $\operatorname{Re}_{c}=420$ is a result of a linear stability analysis which rests on the assumption that the perturbations are infinitesimally small. In RBC, by contrast, the large-scale circulation carries turbulent eddies and temperature fluctuations [23-25] whose amplitude is by no means infinitesimal. (iii) The Tollmien-Schlichting stability analysis assumes that the incoming flow is parallel to the flat plate at which the boundary layer develops. In practice, the large-scale circulation forms an ellipsoid and its angle of attack is not zero with respect to the plate surface. The foregoing observations lead us to the hypothesis that the convective boundary layers in RBC become turbulent much earlier than previously believed. The test of this hypothesis is the central focus of this work. There have been several indications in previous work supporting this hypothesis. It was observed in Ref. [26] for Rayleigh numbers as small as $\mathrm{Ra}=10^{8}$ that significant Reynolds stresses affect the boundary-layer dynamics and that the scaling of the thickness of the velocity boundary layer "does not comply with laminar scaling." Figure 1 in Ref. [20] suggests that the velocity field near the heating plate of $\mathrm{RBC}$ at $\mathrm{Ra}=$ $3 \times 10^{10}$ is far from laminar. These observations have prompted us to examine the boundary layer in turbulent $\mathrm{RBC}$ in more detail using flow visualization, particle image velocimetry, and laser Doppler velocimetry.

The dimensionless parameters that characterize the RB system, a fluid layer of a depth $H$ heated from below and cooled from above, are the Rayleigh number 


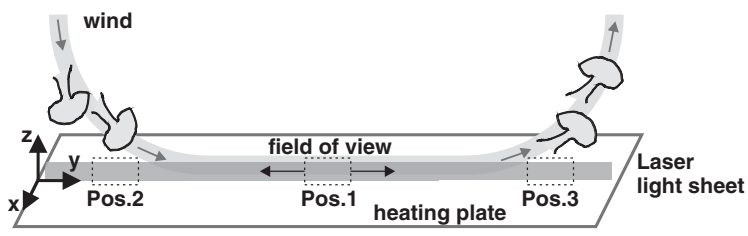

FIG. 1. Experimental setup for the flow visualization in a Rayleigh-Bénard cell of rectangular shape.

$\operatorname{Ra}=\left(\beta g \Delta \vartheta H^{3}\right) /(\nu \kappa)$ and the Prandtl number $\operatorname{Pr}=\nu / \kappa$ for the input as well as the Nusselt number $\mathrm{Nu}=\dot{q}_{c} / \dot{q}_{d}$ and the Reynolds number $\operatorname{Re}=U H / \nu$ for the response of the system. In the case that the fluid layer is laterally confined by sidewalls, the aspect ratio $\Gamma=D / H$ completes the set of parameters. In these definitions variables are assigned to the physical quantities as follows: $\beta$, thermal expansion coefficient; $g$, gravitational acceleration; $\Delta \vartheta$, temperature drop between the heated bottom and the cooled top plate; $\nu$, kinematic viscosity; $\kappa$, thermal diffusivity; $\dot{q}_{c}$, convective heat flux; $\dot{q}_{d}$, diffusive heat flux; $U$, typical mean velocity; $D$, characteristic dimension of the RB cell. Our measurements were performed in a large-scale RB experiment, the Barrel of Ilmenau. This RB cell with a diameter of $D=$ $7.15 \mathrm{~m}$ and a maximum distance $H=6.30 \mathrm{~m}$ between the plates is filled with air and is currently the only one where Ra numbers up to $\mathrm{Ra}=10^{12}$ can be set and the boundary layer is sufficiently large (of the order of $10 \mathrm{~mm}$ ) to probe the flow field with commercial measurement techniques. A detailed description of the facility can be found in Ref. [27]. We acquired flow visualization sequences in a rectangular box of the size $2.5 \times 2.5 \times 0.62 \mathrm{~m}^{3}$ (width $\times$ height $\times$ depth). The specific shape was chosen exclusively for the visualization and the particle image velocimetry measurements. Otherwise the angular dynamics of the wind in cylindrical cells make it impossible to align the laser light sheet with the plane of the wind. The box is made of transparent Perspex and was placed inside the large RB cell using its original heating and cooling plates as bottom and top walls. Since the small rectangular cell is fully surrounded by the larger cylindrical RB cell, $7.15 \mathrm{~m}$ in diameter and $2.50 \mathrm{~m}$ in height, the vertical temperature distribution is almost the same inside and outside the enclosure and a lateral heat exchange throughout the Perspex sidewall is virtually impossible. A vertical laser light sheet of $70 \mathrm{~mm}$ height and about $2 \mathrm{~mm}$ thickness was aligned along the total length of the bottom plate (see Fig. 1). It was generated by a $2 \mathrm{~W}$ cw laser in combination with a specific beam expander that forms a light sheet with accurate parallel lower and upper edges. We took particular care to guide it as close as possible to the surface of the bottom plate and ended up with a maximum gap between the lower edge of the light sheet and the plate surface as small as $0.5 \mathrm{~mm}$ over the entire length of the cell. In order to make the flow visible we added cold-atomized droplets of Di-Ethyl-Hexyl-Sebacat with a typical size of $1 \mu \mathrm{m}$ to the air. Their size is sufficiently small to behave as tracers virtually without any inertia. The motion of the particles is captured in sequences of 1 min duration using a Canon EOS $600 \mathrm{D}$ camera in video mode $(1920$ pixels $\times 1080$ pixels with 30 frames/ sec). The field of view in the plane of the light sheet measures $25 \times 13 \mathrm{~cm}^{2}$. By translating the camera along the side of the cell, visualization sequences were captured along the entire length of the light sheet between $y=0 \mathrm{~m}$ and $y=2.5 \mathrm{~m}$. In this Letter we focus on three selected positions: (i) position 1 at $y=1.25 \mathrm{~m}$, the center of the plate surface, (ii) position 2 at $y=0.45 \mathrm{~m}$, the position where downwelling plumes impinge the plate surface, and (iii) position 3 at $y=2.25 \mathrm{~m}$, the position where the mean wind is deflected by the sidewall and upwelling plumes leave the plate surface.

In Fig. 2, two different snapshots of the flow field [Figs. 2(a) and 2(b)] as well as vector plots of the in-plane velocity field and the vorticity [Figs. 2(c) and 2(d)] above the center of the heating plate (position 1) are shown. The lower black region in both snapshots is the surface of the blank aluminum plate while the white streaks above are the tracks of the particles moving with the flow. The orientation of the mean wind in both images is from left to right. The total height of the light sheet covers a fluid layer of $70 \mathrm{~mm}$ thickness corresponding to $3 \frac{1}{2}$ times the typical thickness of the boundary layer $\delta_{99} \approx 20 \mathrm{~mm}$. Both snapshots have been extracted from a single 1 min sequence separated by only a few seconds and show typical flow states in the fluid layer adjacent to the wall. In order to give the reader a general idea of the near-wall flow field in our experiment, we would like to mention that both the Reynolds number of the outer flow and the shear layer Reynolds number amount to $R e=25.400$ and $\operatorname{Re}_{s}=203$, respectively. In Fig. 2(a) almost all particle tracks are aligned parallel to the horizontal wall. Very close to the plate surface the velocity of the fluid is small and the particles are projected as single dots. The behavior of the particles in this snapshot is typical for a laminar flow field where diffusion dominates the heat transport. It becomes clearer in the movie in the Supplemental Material [28]. As far as we can estimate by visual inspection, this flow behavior could be observed within about $40 \%$ of the total time of the flow visualization sequence at the center of the plate. During the remaining time the flow behaves differently. A representative snapshot of such a flow field is plotted in Fig. 2(b), the corresponding vector plot is shown in Fig. 2(d), and a short sequence is available in the movie in the Supplemental Material [28]. The left-hand part of the snapshot shows a large vortex virtually fully penetrating the boundary layer. This vortex forces the detachment of a fluid parcel roughly as thick as the viscous boundary layer in the right-hand part of the picture. Comparing the specific flow field with, e.g., Prandtl's early flow visualization of the transitional regime in a flat-plate boundary layer [29], a strong similarity is 
(a)
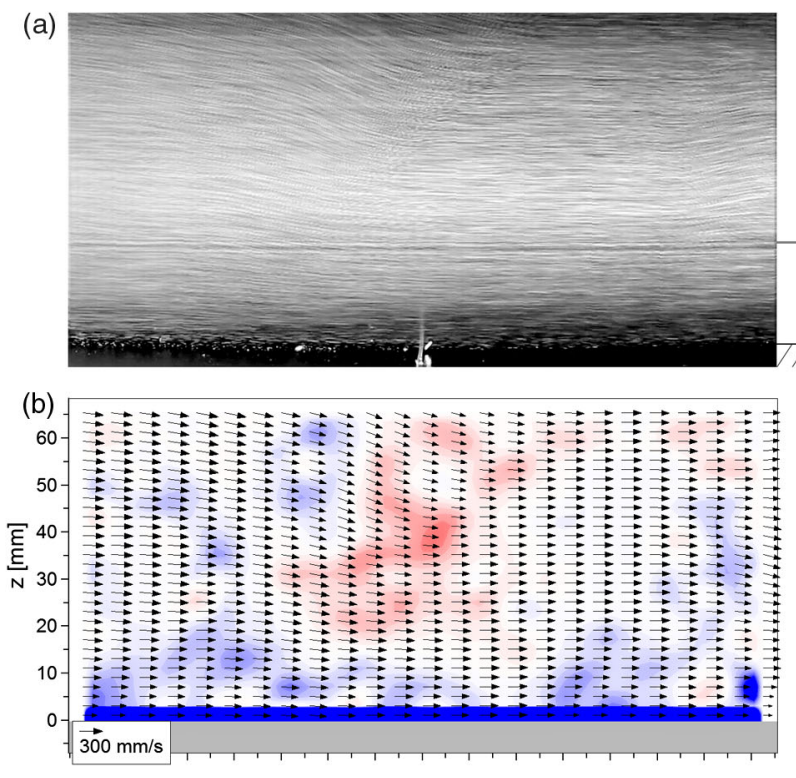
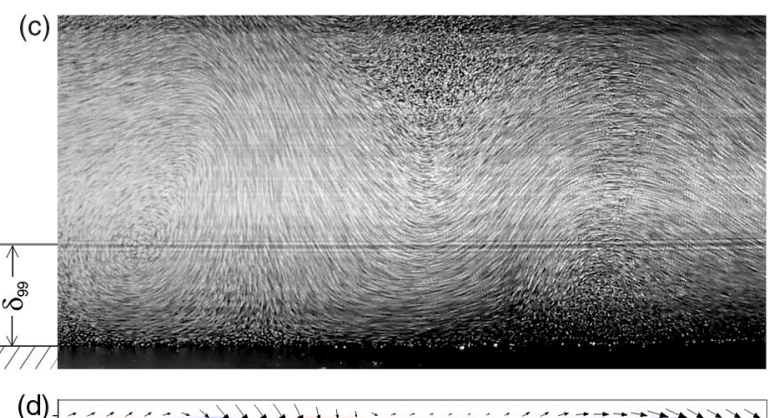

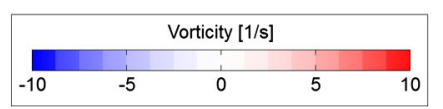

FIG. 2 (color online). Snapshots of contrasting flow states above the center of the heating plate (position 1) separated by only a few seconds. The upper pictures (a),(c) show streaks of droplets of about $1 \mu \mathrm{m}$ size added to the flow, the lower plots (b), (d) show the corresponding vector field, and the color shows the local vorticity.

obvious. There is basically no difference even to a fully developed turbulent boundary (see Ref. [30], e.g., under the entry number 728 or 736). A very remarkable feature of the flow field shown in Fig. 2(d) is the coincidence of clockwise and anticlockwise vorticity inside the boundary layer. This typical behavior of the fully turbulent bulk region is obviously carried over by coherent structures into the boundary layer. The entrainment of turbulent kinetic energy from the mean wind into the boundary layer acts, besides the inner shear and the buoyancy due to the strong wallnormal temperature gradient, as a further mechanism to trigger its transition towards turbulence. The turbulent mixing locally and temporarily enhances the total heat flux throughout the boundary layer with respect to the pure diffusive case. The typical behavior of the flow close to the left and the right sidewall strongly differs from that at the plate center. Snapshot taken at position $2(y=0.45 \mathrm{~m})$ and position $3(y=2.37 \mathrm{~m})$ are shown in Figs. 3(a) and 3(c). Figure 3(a) shows a representative flow picture observed at the specific flow region where cold plumes that drop down from the cooled top plate hit the surface of the heated bottom plate (see Fig. 1). In Fig. 3(b), the corresponding vector plot obtained from two subsequent snapshots with a time separation of $\tau=33 \mathrm{~ms}$ is shown. In particular, the flow in the right half of the flow domain is clearly oriented towards the wall. When the fluid impinges the surface of the plate it generates eddies like on the left with respect to the stagnation point or even a total recirculation as is visible in the upper left region of the plot. A more comprehensive insight into the dynamics of the boundary-layer flow field in this specific flow region is provided in the movie in the Supplemental Material [28], which shows an $8 \mathrm{sec}$ sequence of the flow visualization. The sequence clearly demonstrates the permanent turbulent mixing of the boundary layer (except the viscous sublayer) and is in good agreement with work recently reported by Ahlers et al. [11]. This causes an enhanced heat transport between the plate surface and the fluid with respect to the central region of the plate. The local nonuniformity of the wall-heat flux has been evaluated by measurements using an infrared camera and a special coating of the plate surface [31]. These measurements show that the local, time-averaged heat flux at this specific surface region exceeds the global one by about $20 \%$. An enhancement of the heat flux can be observed as well at the opposite sidewall where the plumes detach from the boundary layer (position 3, $y=2.25 \mathrm{~m}$ ).

In order to quantify the effect of turbulence in the close vicinity of the surface of the horizontal plates, we analyzed highly resolved, local measurements of all three velocity components using laser Doppler velocimetry (see Ref. [32] for detailed information). The measurements were undertaken in a RB cell of cylindrical shape with $\Gamma=1.0$ and $\mathrm{Ra}=1.5 \times 10^{10}$, the same set of parameters as adjusted in the rectangular cell. We note here that we used these measurements for the evaluation since the measurement time for each of the data points amounted to $1 \mathrm{hr}, 60$ times longer than the duration of the visualization sequences. We computed the profile of the turbulence intensity $I\left(z^{+}\right)$along the central axis of the RB cell according to the following definition: 
(a)

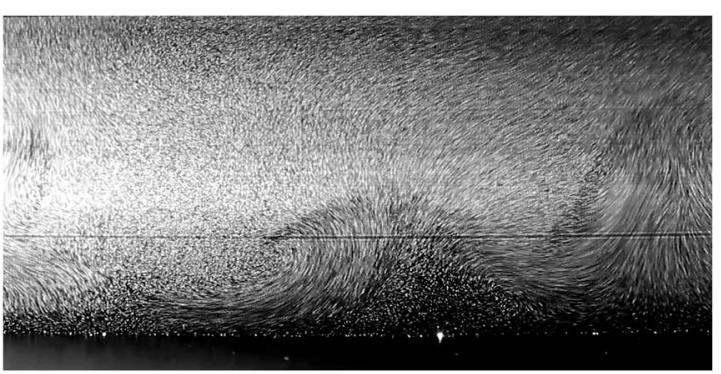

(b)

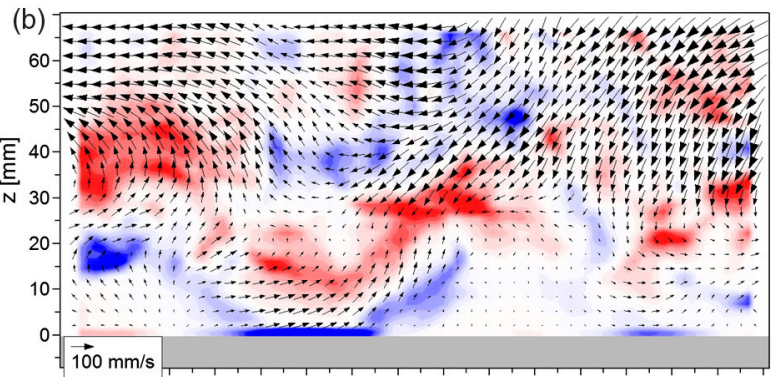

(c)

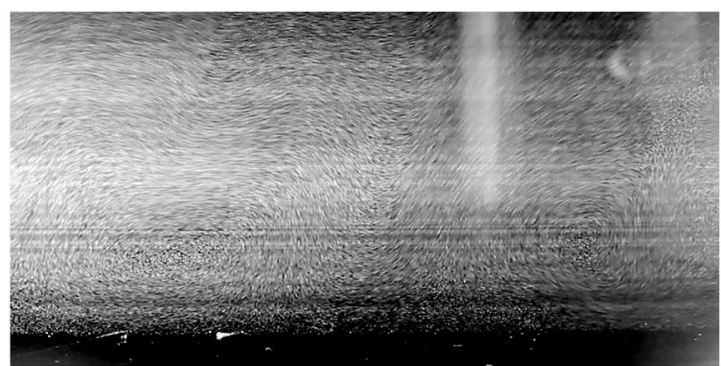

(d)

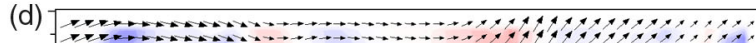

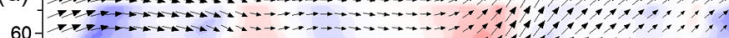

$50-$

ह 20

$2+\ldots+\ldots$
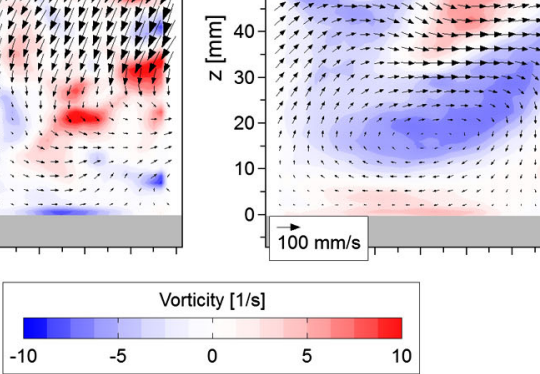

FIG. 3 (color online). Flow field at position 2 (a),(b) and position 3 (c),(d) close to the left and the right sidewall, respectively. The upper pictures show streaks of small droplets of about $1 \mu \mathrm{m}$ size added to the flow, the lower plots show the corresponding vector field as well as the local vorticity.

$$
I\left(z^{+}\right)=\frac{U^{\prime}\left(z^{+}\right)}{\bar{U}_{\max }}=\frac{\sqrt{1 / 3\left[u^{\prime 2}+v^{\prime 2}+w^{\prime 2}\right]}}{\sqrt{\bar{u}^{2}+\bar{v}^{2}+\bar{w}^{2}}},
$$

where $u^{\prime}, v^{\prime}$, and $w^{\prime}$ denote the fluctuations of the $x, v$, and $w$ components of the local velocity vector and $\bar{u}, \bar{v}$, and $\bar{w}$ stand for their mean at the $z$ position of the maximum horizontal velocity. The $z^{+}$coordinate is defined as $z^{+}=z u_{\tau} / \nu$. The turbulence intensity represents a very common quantity in fluid dynamics to characterize the amount of turbulent kinetic energy contained in a flow. We mention two typical examples. In the atmospheric boundary layer the turbulence intensity amounts to $I \approx 0.2$ [33], and in a fully developed pipe flow at $\operatorname{Re} \approx 10.000$, it is $I \approx 0.5$. Our results $I\left(z^{+}\right)$are plotted in Fig. 4 . At the

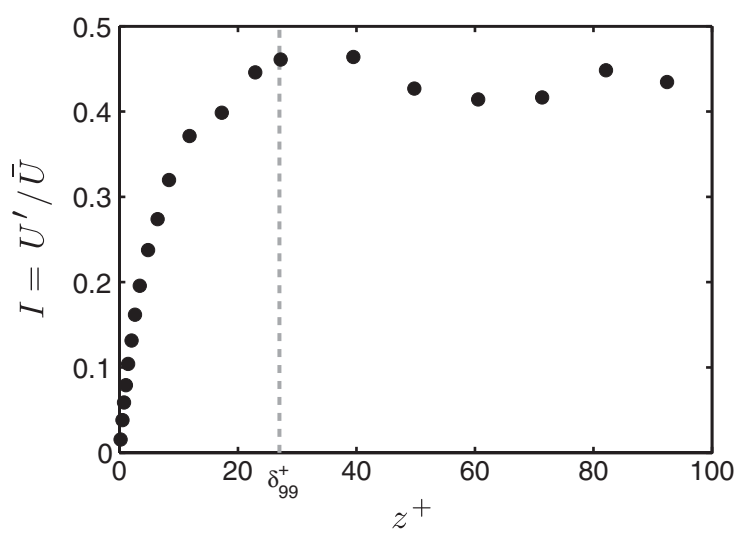

FIG. 4. Profile of the turbulence intensity $I\left(z^{+}\right)$. The dashed line indicates the boundary layer thickness $\delta_{99}^{+}=\delta_{99} u_{\tau} / \nu$. dimensionless wall distance $z^{+}=5$, which is usually considered as the outer edge of the viscous sublayer, the turbulence intensity already amounts to $I(5)=0.23$, comparable with the level in the atmospheric boundary layer, and it achieves its maximum $I=0.46$ right at the outer limit of the boundary layer at $z^{+}=27$, corresponding to $z=19.8 \mathrm{~mm}$.

Summarizing our results, for the first time a direct experimental evidence of turbulence in the boundary layer in Rayleigh-Bénard convection has been provided. It could be demonstrated that turbulence sets in locally at Rayleigh numbers as low as $\mathrm{Ra}=1.4 \times 10^{10}\left(\operatorname{Re}_{s} \approx 200\right)$, which is significantly below the critical limit of $\mathrm{Ra}_{c} \approx 10^{14}$ $\left(\operatorname{Re}_{s, c} \approx 420\right)$ as predicted by Grossmann and Lohse [14]. The onset of turbulence is triggered by coherent structures carrying turbulent kinetic energy from the bulk into the near-wall flow region. This could be identified as a second mechanism to mix the boundary layers in addition to their destabilization due to the inner shear. Analyzing local 3D velocity measurements using laser Doppler anemometry, we found that, even at the moderate $\mathrm{Ra}$ number of $\mathrm{Ra}=1.4 \times 10^{10}$, the turbulence intensity inside the boundary layers is of the same order as compared with the atmospheric boundary layer or a turbulent pipe flow.

We wish to acknowledge the Deutsche Forschungsgemeinschaft under Grant No. PU 436/1-1, the European Union under the COST program MP0806, as well as the Thueringer Ministerium fuer Wissenschaft, Forschung und Kunst for financial support of the work reported here. 
*ronald.dupuits@tu-ilmenau.de

[1] P. F. Linden, Annu. Rev. Fluid Mech. 31, 201 (1999).

[2] R. F. Stein and A. Nordlund, Astrophys. J. 342, L95 (1989).

[3] A. Ganachaud and C. Wunsch, Nature (London) 408, 453 (2000).

[4] E. D. Siggia, Annu. Rev. Fluid Mech. 26, 137 (1994).

[5] E. Bodenschatz, W. Pesch, and G. Ahlers, Annu. Rev. Fluid Mech. 32, 709 (2000).

[6] D. Lohse and K.-Q. Xia, Annu. Rev. Fluid Mech. 42, 335 (2010).

[7] G. Ahlers, S. Grossmann, and D. Lohse, Rev. Mod. Phys. 81, 503 (2009).

[8] R.-H. Kraichnan, Phys. Fluids 5, 1374 (1962).

[9] J.-J. Niemela, J. Fluid Mech. 481, 355 (2003).

[10] P.-E. Roche, F. Gauthier, R. Kaiser, and J. Salort, New J. Phys. 12, 085014 (2010).

[11] G. Ahlers, E. Bodenschatz, D. Funfschilling, S. Grossmann, X. He, D. Lohse, R. J. A. M. Stevens, and R. Verzicco, Phys. Rev. Lett. 109, 114501 (2012).

[12] P. Urban, V. Musilová, and L. Skrbek, Phys. Rev. Lett. 107, 014302 (2011).

[13] S. Grossmann and D. Lohse, J. Fluid Mech. 407, 27 (2000).

[14] S. Grossmann and D. Lohse, Phys. Rev. E 66, 016305 (2002).

[15] W. Tollmien, 1. Mitteilung, Nachr. Ges. Wiss. Goettingen, Math. Phys. Klasse, 21 (1929).

[16] H. Schlichting, Nachr. Ges. Wiss. Goettingen, Math. Phys. Klasse, 181 (1933).

[17] K. Stewartson, Z. Angew Math. Phys. 9a, 276 (1958).
[18] Z. Rotem and L. Claassen, J. Fluid Mech. 39, 173 (1969).

[19] R. du Puits, C. Resagk, and A. Thess, Phys. Rev. Lett. 99, 234504 (2007).

[20] N. Shi, M. S. Emran, and J. Schumacher, J. Fluid Mech. 706, 5 (2012).

[21] J. D. Scheel, E. Kim, and R. K. White, J. Fluid Mech. 711, 281 (2012).

[22] O. Shishkina and A. Thess, J. Fluid Mech. 633, 449 (2009).

[23] C. Sun, K.-Q. Xia, and P. Tong, Phys. Rev. E 72, 026302 (2005).

[24] Q. Zhou and K.-Q. Xia, Phys. Rev. Lett. 104, 104301 (2010).

[25] G. Silano, K. R. Sreenivasan, and R. Verzicco, J. Fluid Mech. 662, 409 (2010).

[26] M. van Reeuwijk, H. J. J. Jonker, and K. Hanjalić, Phys. Rev. E 77, 036312 (2008).

[27] R. du Puits, C. Resagk, and A. Thess, New J. Phys. 15, 013040 (2013).

[28] See Supplemental Material at http://link.aps.org/ supplemental/10.1103/PhysRevLett.112.124301 for visualization of the flow field according to Figs. 2a,c and 3a,c.

[29] L. Prandtl, Z. VDI 77, 105 (1933).

[30] G. M. Homsy, Multimedia Fluid Mechanics (Cambridge University Press, Cambridge, England, 2007).

[31] R. Kaiser and R. du Puits (to be published).

[32] L. Li, N. Shi, R. du Puits, C. Resagk, J. Schumacher, and A. Thess, Phys. Rev. E 86, 026315 (2012).

[33] J. Counihan, Atmos. Environ. 9, 871 (1975). 